\title{
A rare incidence of averting 'maternal near-miss' in a case of spontaneous uterine rupture in shock
}

\author{
Jayanta K. Biswas ${ }^{1 *}$, Sanjaya K. Gupta ${ }^{2}$
}

\begin{abstract}
${ }^{1}$ Department of Obstetrics and Gynecology, Military Hospital, Namkum, Ranchi, Jharkhand, India
${ }^{2}$ Department of Anaesthesiology and Critical care, Military Hospital, Pathankot, Punjab, India
\end{abstract}

Received: 06 December 2021

Accepted: 04 January 2022

\section{*Correspondence:}

Dr. Jayanta K. Biswas,

E-mail: jayantabiswas1977@gmail.com

Copyright: ( ) the author(s), publisher and licensee Medip Academy. This is an open-access article distributed under the terms of the Creative Commons Attribution Non-Commercial License, which permits unrestricted non-commercial use, distribution, and reproduction in any medium, provided the original work is properly cited.

\begin{abstract}
Uterine rupture is a rare but catastrophic obstetric emergency associated with significant feto-maternal morbidity and mortality. There are various risk factors and wide spectrum of clinical presentations have been identified; previous cesarean delivery being most common risk factor. Spontaneous rupture of unscarred uterus is infrequently encountered, but very dangerous for both mother and fetus as remotely suspected. Usually, spontaneous rupture of primigravida uterus is considered almost immune as it is rare but not unheard of; it may occur before or after onset of labour, at term or preterm and with or without fetal heart rate abnormality. The reported case was such a case of spontaneous unscarred uterine rupture with favourable outcome for both mother and baby.
\end{abstract}

Keywords: Unscarred uterus, Spontaneous uterine rupture, Favourable pregnancy outcome

\section{INTRODUCTION}

Uterine rupture in pregnancy is a rare event but can frequently result in life threatening maternal and fetal compromise; even maternal and perinatal mortality ranges between $1-13 \%$ and $74-92 \%$ respectively. ${ }^{1}$

It can happen either in unscarred or previously scarred uterus. Previous uterine scar is a well-known risk factor for uterine rupture, but the normal unscarred uterus is least susceptible to rupture with overall incidence being $0.0033 \%$. $^{2}$ However, its incidence is still higher in developing countries. ${ }^{3}$

Major risk factors for rupture of unscarred uterus are multiparity, congenitally anomalous uterus, obstructed labour, morbidly adhered placenta, injudicious use of uterotonics and rarely intrauterine manipulations. ${ }^{1}$ Clinical presentation of spontaneous uterine rupture is highly variable with nonspecific maternal complaints and fetal status, requiring high index of suspicion. ${ }^{4}$ Maternal tachycardia, fetal distress and bleeding per vaginum are most common presentation. ${ }^{5}$

Perinatal and maternal outcomes are depending on risk factor assessment, early recognition of clinical signs and symptoms and also prompt surgical intervention. ${ }^{6}$ The case reported was a promptly managed spontaneous uterine rupture in latent labour with favourable maternal and perinatal outcome.

\section{CASE REPORT}

A 27 years old booked antenatal case with no living issue and history of single spontaneous abortion at early first trimester without any medical or surgical intervention in previous pregnancy; with no co-morbidity admitted in latent labour at 39 weeks gestation.

Her blood group was 'A- positive' and her all relevant antenatal investigations were essentially normal. On admission, she was in latent labour with all admission tests 
within normal limits and she was kept on feto-maternal monitoring as per departmental policy.

Suddenly she had developed tachycardia, marginal hypotension associated with continuous fetal variable deceleration. She also had abdominal pain without any signs of abruptio placentae or any abdominal swelling. Immediate decision for performing Lower segment cesarean section delivery (LSCS) taken with due counselling and written consent in view of fetal distress and ensuing shock- possibly with spontaneous uterine rupture. LSCS revealed massive hemoperitoneum; patient was in class-II hypovolemic shock. Urgent delivery was done through Kerr's incision on lower uterine segment (LUS), delivering an alive healthy female child with normal APGAR score. Source of haemorrhage was found to be from fundal uterine rupture site of $8 \mathrm{~cm}$ in length (Figure 1).

Closure of LUS scar along with repair of fundal uterine rupture site was performed satisfactorily (Figure 2). Simultaneously, patient was resuscitated with intravenous fluids and noradrenaline infusion to maintain mean arterial pressure $(\mathrm{MAP})>65 \mathrm{~mm}$ of $\mathrm{Hg}$ as there was sudden severe hypotension with tachycardia during intra-operative period. 2 units of whole blood was transfused during intraoperative and post-operative periods. Post-operative recovery was uneventful.

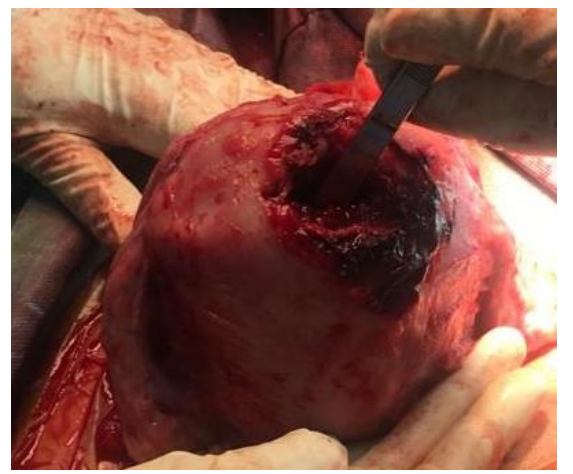

Figure 1: Fundal uterine rupture site indicated after LSCS delivery.

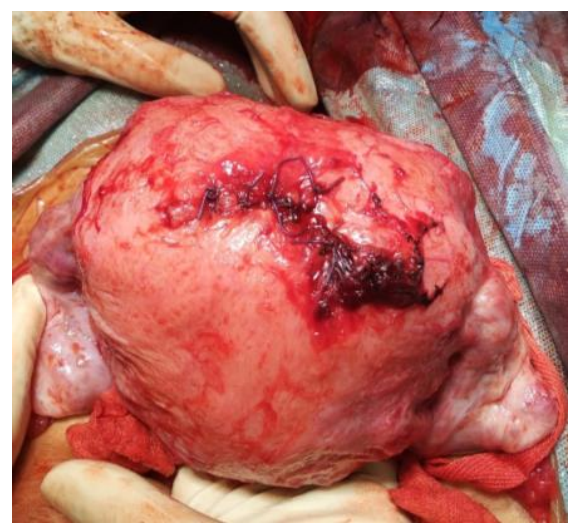

Figure 2: Uterus following repair of fundal rupture site.

\section{DISCUSSION}

Uterine rupture in pregnancy is rare but can lead to catastrophic obstetric event with life-threatening maternal and fetal complications. Collective data from metaanalysis of published literature indicated overall incidence of $0.07 \%$ and that of spontaneous uterine rupture in unscarred uterus in developed countries was $0.012 \%$; whereas there was increased incidence in developing countries $(0.11 \%)$ mainly attributable to higher incidence of neglected obstructed labour.

The anatomically normal unscarred uterus is least susceptible for rupture, though some predisposing factors are found like- advanced maternal age, multiparity, neglected labour with or without injudicious use of uterotonics, fetal malpresentation, fetal macrosomia, breech extraction and uterine instrumentation. ${ }^{7}$ In the case reported no such predisposing factor was present.

Clinical presentations are varied and mainly depends on the timing of its presentation and the site and extent of rupture. Typically rupture in unscarred uterus is more dangerous than in scarred rupture due to high vascularity. ${ }^{7}$ Classical signs and symptoms are loss of uterine contractility, abdominal pain, fetal distress, haemorrhage and shock; prolonged fetal heart rate decelerations are often first and only signs as per Leung et al. ${ }^{8}$ The case presented also had similar presentations.

Early definitive intervention can avoid major fetal and maternal morbidity and mortality; studies also have indicated delivery within 10-37 min of rupture is necessary to prevent serious fetal complications like fetal hypoxia, acidosis, neonatal intensive care admissions and even death. ${ }^{8}$ Maternal complications includes excessive blood loss leading to hypovolemic shock, maternal bladder injury, needs for cesarean hysterectomies and even maternal death. ${ }^{9}$

After delivery of fetus, definitive surgical treatment for mother is done depending on type and extent of rupture, general condition and degree of haemorrhage and also mother's desire for future child-bearing. Hysterectomy should be considered treatment of choice when repair of rupture site cannot be contemplated. ${ }^{10}$ Uterine rupture site was successfully repaired in the case reported.

\section{CONCLUSION}

Spontaneous unscarred uterine rupture in pregnancy is rare event but often life-threatening to both fetus and mother even with varied clinical presentations. Perinatal outcomes can be optimized by awareness, early recognition and prompt intervention to avoid deadly feto-maternal morbidity and mortality.

Funding: No funding sources

Conflict of interest: None declared

Ethical approval: Not required 


\section{REFERENCES}

1. Hofmeyr GJ, Say L, Gülmezoglu AM. WHO systematic review of maternal mortality and morbidity: the prevalence of uterine rupture. BJOG. 2005;112(9):1221-8.

2. Gardeil F, Daly S, Turner MJ. Uterine rupture in pregnancy reviewed. Eur J Obstet Gynecol Reprod Biol. 1994;56(2):107-10.

3. Turner MJ. Uterine rupture. Best Pract Res Clin Obstet Gynaecol. 2002;16(1):69-79.

4. Dow M, Wax JR, Pinette MG, Blackstone J, Cartin A. Third-trimester uterine rupture without previous cesarean: a case series and review of the literature. Am J Perinatol. 2009;26(10):739-44.

5. Mazzone ME, Woolever J. Uterine rupture in a patient with an unscarred uterus: a case study. WMJ. 2006;105(2):64-6.

6. Miller DA, Goodwin TM, Gherman RB, Paul RH. Intrapartum rupture of the unscarred uterus. Obstet Gynecol. 1997;89(5):671-3.
7. Nahum GG, Pham KQ. Uterine rupture in pregnancy, 2018. Available at: https://reference.medscape.com/article/275854overvi ew\#showall. Accessed on 28 November 2021.

8. Leung AS, Leung EK, Paul RH. Uterine rupture after previous cesarean delivery: maternal and fetal consequences. Am J Obstet Gynecol. 1993;169(4):945-50.

9. Chuni N. Analysis of uterine rupture in a tertiary center in Eastern Nepal: lessons for obstetric care. J Obstet Gynaecol Res. 2006;32(6):574-9.

10. Kapoor DS, Sharma SD, Alfirevic Z. Management of unscarred ruptured uterus. $J$ Perinat Med. 2003;31(4):337-9.

Cite this article as: Biswas JK, Gupta SK. A rare incidence of averting 'maternal near-miss' in a case of spontaneous uterine rupture in shock. Int J Reprod Contracept Obstet Gynecol 2022;11:617-9. 